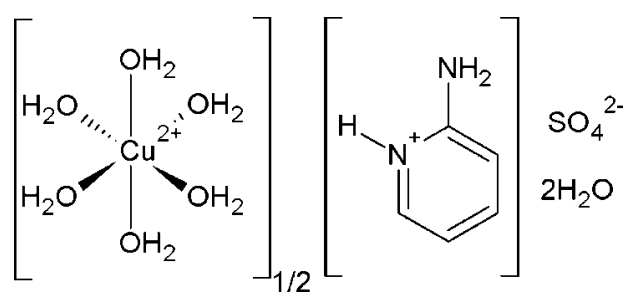

\section{Crystal structure of new organically templated copper sulfate with 2-amino- pyridinium}

\section{Tamara J. Lukianova,* Vasyl Kinzhybalo and Adam Pietraszko}

Institute of Low Temperature and Structure Research, Polish Academy of Sciences, Okolna str. 2, PO Box 1410, 50-950 Wroclaw, Poland. *Correspondence e-mail:

T.Lukianova@int.pan.wroc.pl

Received 27 September 2015; accepted 5 October 2015

Edited by E. F. C. Herdtweck, Technischen Universität München, Germany

The title compound, $\left(\mathrm{C}_{5} \mathrm{H}_{7} \mathrm{~N}_{2}\right)_{2}\left[\mathrm{Cu}\left(\mathrm{H}_{2} \mathrm{O}\right)_{6}\right]\left(\mathrm{SO}_{4}\right)_{2} \cdot 4 \mathrm{H}_{2} \mathrm{O}$ [systematic name: bis(2-aminopyridinium) hexaaquacopper(II) bis(sulfate) tetrahydrate], comprises axially elongated hexaaqua-coordinated octahedral $\mathrm{Cu}^{\mathrm{II}}$ ions located on an inversion centre, non-coordinating sulfate anions, 2-aminopyridinium cations and lattice water molecules. The crystal structure is built of successive inorganic and organic layers extending parallel to (001) that are connected by an extensive three-dimensional hydrogen-bonded network of the type $\mathrm{O}-$ $\mathrm{H} \cdots \mathrm{O}$ and $\mathrm{N}-\mathrm{H} \cdots \mathrm{O}$, as well as $\pi-\pi$ interactions [centroidcentroid distance 3.4140 (14) $\AA$, offset $0.277 \AA$ ]

Keywords: crystal structure; organically templated materials; 2-aminopyridine; sulfates; hydrogen bonding; $\pi-\pi$ interactions.

CCDC reference: 1429506

\section{Related literature}

For applications of 2-aminopyridine, see: Windholz (1976). For 2-aminopyridinium sulfate, see: Jebas et al. (2006). For other compounds with copper(II), see: Naili et al. (2006); Rekik et al. (2006).

\subsection{Data collection \\ Rigaku Oxford Diffraction Diffraction, 2015) \\ Xcalibur, Sapphire2 diffractometer \\ Absorption correction: multi-scan (CrysAlis PRO; Rigaku Oxford

$$
T_{\min }=0.720, T_{\max }=1.000
$$ 7926 measured reflections 3173 independent reflections 2268 reflections with $I>2 \sigma(I)$ $R_{\text {int }}=0.038$}

2.3. Refinement

$R\left[F^{2}>2 \sigma\left(F^{2}\right)\right]=0.044$

$w R\left(F^{2}\right)=0.094$

$S=1.03$

3173 reflections

160 parameters

$\mathrm{H}$-atom parameters constrained

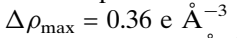

$\Delta \rho_{\min }=-0.41{\mathrm{e} \AA^{-3}}^{-3}$

Table 1

Hydrogen-bond geometry $\left(\AA,^{\circ}\right)$.

\begin{tabular}{lllll}
\hline$D-\mathrm{H} \cdots A$ & $D-\mathrm{H}$ & $\mathrm{H} \cdots A$ & $D \cdots A$ & $D-\mathrm{H} \cdots A$ \\
\hline $\mathrm{O} 11 W-\mathrm{H} 11 A \cdots \mathrm{O} 14 W^{\mathrm{i}}$ & 0.84 & 2.00 & $2.821(3)$ & 167 \\
$\mathrm{O} 11 W-\mathrm{H} 11 B \cdots \mathrm{O} 12^{\mathrm{ii}}$ & 0.84 & 2.22 & $3.032(4)$ & 162 \\
$\mathrm{O} 12 W-\mathrm{H} 12 A \cdots \mathrm{O} 15 W$ & 0.84 & 1.88 & $2.719(3)$ & 172 \\
$\mathrm{O} 12 W-\mathrm{H} 12 B \cdots \mathrm{O} 13$ & 0.84 & 1.85 & $2.677(3)$ & 171 \\
$\mathrm{O} 13 W-\mathrm{H} 13 A \cdots \mathrm{O} 14^{\mathrm{iii}}$ & 0.84 & 1.90 & $2.733(3)$ & 174 \\
$\mathrm{O} 13 W-\mathrm{H} 13 B \cdots \mathrm{O} 14 W$ & 0.84 & 1.88 & $2.706(3)$ & 168 \\
$\mathrm{~N} 1-\mathrm{H} 1 \cdots \mathrm{O} 13$ & 0.86 & 2.03 & $2.855(3)$ & 160 \\
$\mathrm{~N} 2-\mathrm{H} 2 A \cdots \mathrm{O} 11$ & 0.86 & 2.01 & $2.869(3)$ & 176 \\
$\mathrm{~N} 2-\mathrm{H} 2 B \cdots \mathrm{O} 12^{\text {iv }}$ & 0.86 & 2.05 & $2.914(3)$ & 178 \\
$\mathrm{O} 14 W-\mathrm{H} 14 A \cdots \mathrm{O} 15 W^{\text {ii }}$ & 0.84 & 1.92 & $2.758(3)$ & 174 \\
$\mathrm{O} 14 W-\mathrm{H} 14 B \cdots \mathrm{O} 14^{\mathrm{ii}}$ & 0.84 & 1.90 & $2.738(3)$ & 176 \\
$\mathrm{O} 15 W-\mathrm{H} 15 A \cdots \mathrm{O} 11^{\mathrm{v}}$ & 0.84 & 1.93 & $2.761(3)$ & 169 \\
$\mathrm{O} 15 W-\mathrm{H} 15 B \cdots \mathrm{O} 12^{\mathrm{ii}}$ & 0.84 & 1.93 & $2.760(3)$ & 170 \\
\hline
\end{tabular}

Symmetry codes: (i) $-x,-y+1,-z+1$; (ii) $-x,-y,-z+1$; (iii) $x, y+1, z$; (iv) $-x+1,-y,-z+2 ;(\mathrm{v})-x+1,-y,-z+1$.

Data collection: CrysAlis PRO (Rigaku Oxford Diffraction, 2015); cell refinement: CrysAlis PRO; data reduction: CrysAlis PRO; program(s) used to solve structure: SHELXS2014/7 (Sheldrick, 2008); program(s) used to refine structure: SHELXL2014/7 (Sheldrick, 2015); molecular graphics: DIAMOND (Brandenburg et al., 1997); software used to prepare material for publication: OLEX2 (Dolomanov et al., 2009). 


\section{Acknowledgements}

This research was supported by an ILT\&SR PAS grant for young scientists and $\mathrm{PhD}$ students funded by the Ministry of Science and Higher Education of Poland.

Supporting information for this paper is available from the IUCr electronic archives (Reference: HP2072).

\section{References}

Brandenburg, K. (1997). DIAMOND. Crystal Impact GbR, Bonn, Germany.
Dolomanov, O. V., Bourhis, L. J., Gildea, R. J., Howard, J. A. K. \& Puschmann, H. (2009). J. Appl. Cryst. 42, 339-341.

Jebas, S. R., Balasubramanian, T., Peschar, R. \& Fraanje, J. (2006). Acta Cryst. E62, o2606-02607.

Naili, H., Rekik, W., Bataille, T. \& Mhiri, T. (2006). Polyhedron, 25, 3543-3554.

Rekik, W., Naili, H., Bataille, T., Roisnel, T. \& Mhiri, T. (2006). Inorg. Chim. Acta, 359, 3954-3962.

Rigaku Oxford Diffraction (2015). CrysAlis PRO. Rigaku Oxford Diffraction, Yarnton, England.

Sheldrick, G. M. (2008). Acta Cryst. A64, 112-122.

Sheldrick, G. M. (2015). Acta Cryst. C71, 3-8.

Windholz, M. (1976). The Merck Index, 9th ed. Boca Raton, USA: Merck \& Co. Inc. 


\section{supporting information}

Acta Cryst. (2015). E71, m191-m192［https://doi.org/10.1107/S2056989015018629]

\section{Crystal structure of new organically templated copper sulfate with 2-amino- pyridinium}

\section{Tamara J. Lukianova, Vasyl Kinzhybalo and Adam Pietraszko}

\section{S1. Comment}

Crystal structure of I is composed of 2-aminopyridinium (2ap) cations, isolated sulfate anions, metal cations octahedrally coordinated by six water molecules $\left[\mathrm{Cu}\left(\mathrm{H}_{2} \mathrm{O}\right)_{6}\right]^{2+}$ and uncoordinated water molecules. The atom labeling scheme for compound $\mathbf{I}$ is shown in Fig. 1. The asymmetric unit contains one half of $\mathrm{Cu}$ atom (lies on a center of inversion) along with three water molecules coordinated to it, one sulfate group, one protonated amine and two solvation water molecules. The $\mathrm{Cu}$ ion environment shows considerable axial deformation to tetragonal bipyramidal due to Jahn-Teller effect. The $\mathrm{Cu}-\mathrm{O} 12 \mathrm{~W}$ and $\mathrm{Cu}-\mathrm{O} 13 \mathrm{~W}$ distances are equal to 1.935 (2) and 1.9790 (18) $\AA$, respectively, and the $\mathrm{Cu}-\mathrm{O} 11 \mathrm{~W}$ distance is strongly elongated to 2.398 (2) $\AA$. The distances within the $\left[\mathrm{Cu}\left(\mathrm{H}_{2} \mathrm{O}\right)_{6}\right]^{2+}$ octahedron are comparable to those observed in other compounds (Naïli et al., 2006; Rekik et al., 2006). The crystal packing consists of successive organic and inorganic layers parallel to $0 x y$ plane. Inorganic layers are stabilized by a series of $\mathrm{O}-\mathrm{H} \cdots \mathrm{O}$ hydrogen bonds (Table 1 and Fig. 2). Organic layers are built of $\pi$ - $\pi$ interacting stacks of 2ap cations $(\mathrm{Cg} \cdots \mathrm{Cg} 3.4140$ (14) $\AA$, offset $0.277 \AA$ ) connected to inorganic layers through $\mathrm{N}-\mathrm{H} \cdots \mathrm{O}$ hydrogen bonds (Table 1 and Fig. 3).

\section{S2. Experimental}

The title compound was synthesized by the following method. 2-aminopyridine $(0.19 \mathrm{~g}, 2 \mathrm{mmol})$ was dissolved in $4 \mathrm{ml}$ double distilled water to obtain solution $\mathrm{A}$. The $\mathrm{pH}$ of the solution was adjusted to 2.5 , by the addition of $30 \%$ sulfuric acid. Copper sulfate $(0.149 \mathrm{~g}, 6 \mathrm{mmol})$ was dissolved in $3 \mathrm{ml}$ double distilled water to obtain solution B. Solution A was added on solution B. The resulting solution was kept at room temperature. The green crystals of the title compound were obtained by slow evaporation during the period of several months.

\section{S3. Refinement}

The $\mathrm{H}$ atoms of water molecules were located from difference Fourier maps and were refined with $\mathrm{O}-\mathrm{H}$ distances restrained to $0.840(2) \AA$ and $\operatorname{Uiso}(\mathrm{H})=1.5 \mathrm{Ueq}(\mathrm{O})$. In final refinement cycles $\mathrm{H}$ atoms of water were let to ride on parent $\mathrm{O}$ atom (AFIX 3). 


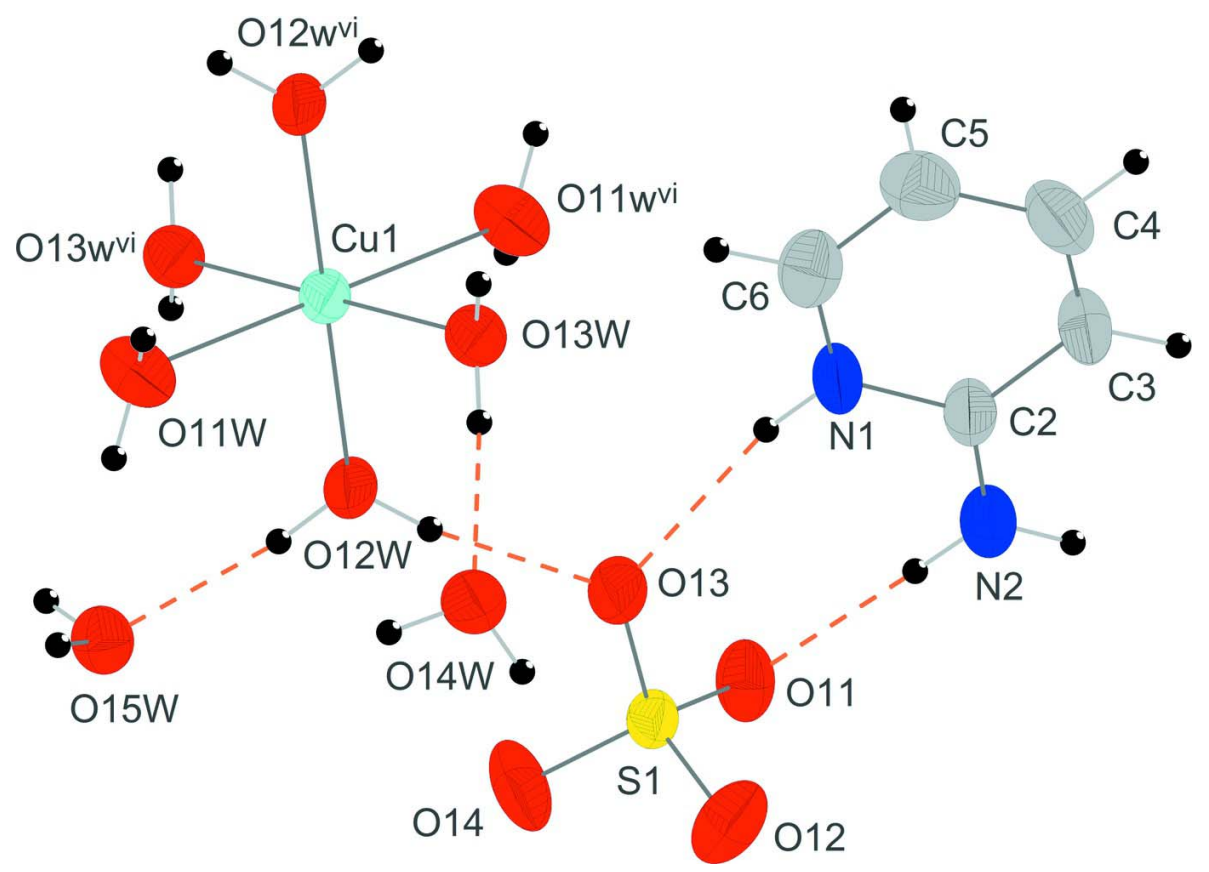

Figure 1

The asymmetric unit of the title compound, showing the crystallographic numbering scheme. Displacement ellipsoids are drawn at the $50 \%$ probability level. Hydrogen bonds are denoted by orange dashed lines. [Symmetry codes: $(v i)-x+1,-y$ $+1,-z+1]$. 


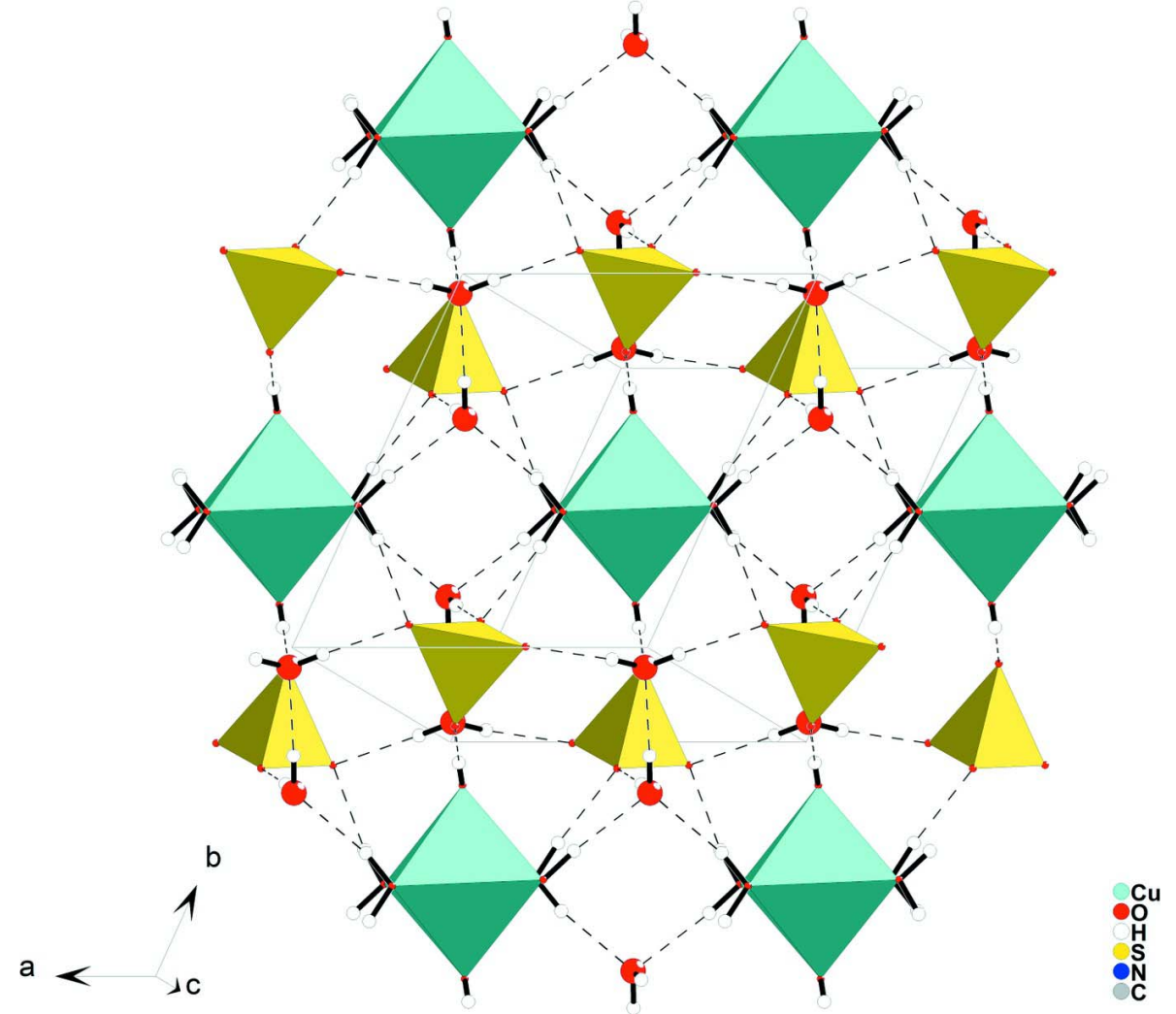

Figure 2

View of inorganic layers along perpendicular to this layer direction $\left(\mathrm{c}^{*}\right)$. Dashed lines indicate the hydrogen bonds. 


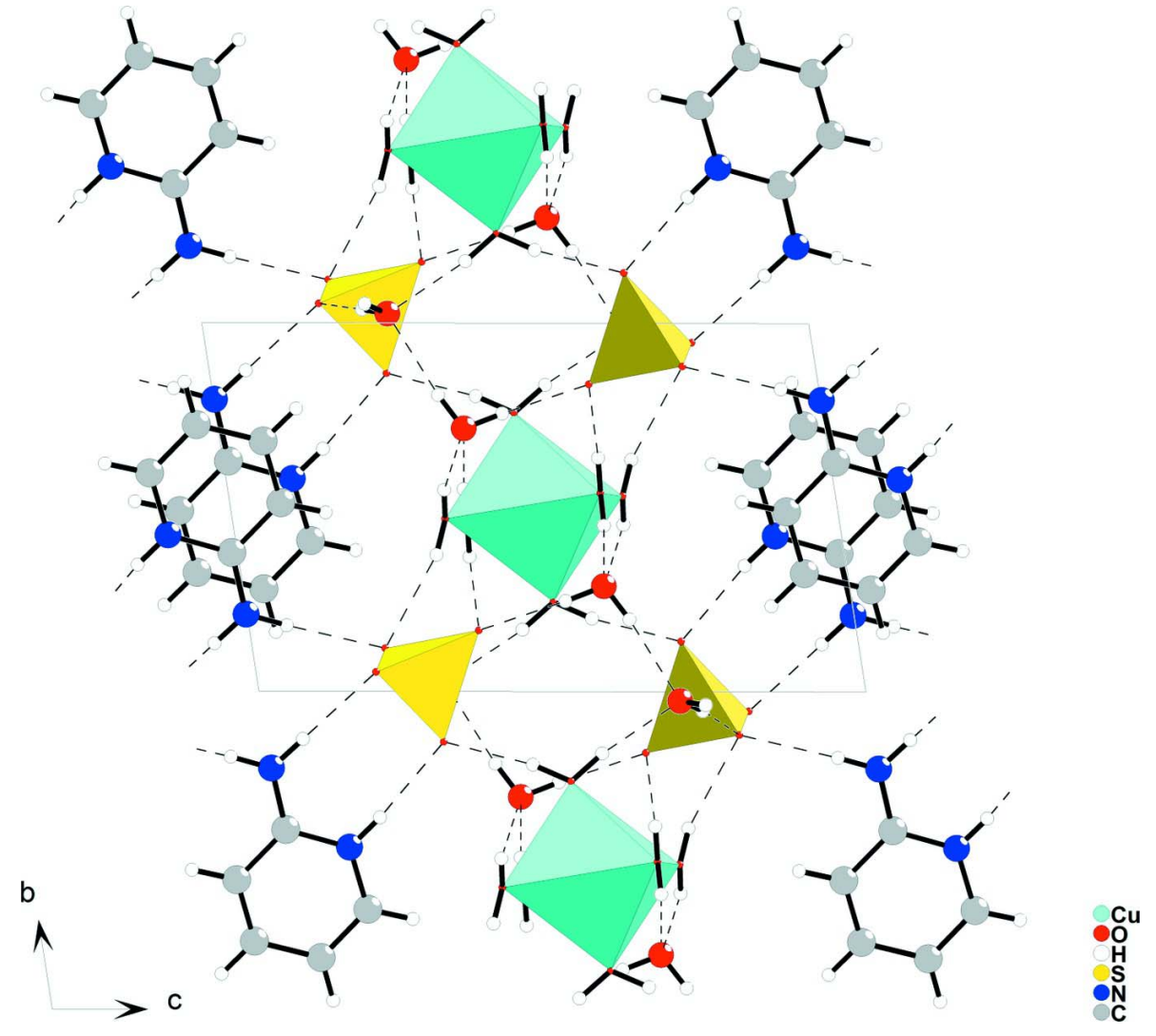

Figure 3

The molecular arrangement in $\left(\mathrm{C}_{5} \mathrm{H}_{7} \mathrm{~N}_{2}\right)_{2}\left[\mathrm{Cu}^{\mathrm{II}}\left(\mathrm{H}_{2} \mathrm{O}\right)_{6}\right]\left(\mathrm{SO}_{4}\right)_{2} \cdot 4 \mathrm{H}_{2} \mathrm{O}$ viewed along [100]. Dashed lines represent hydrogen bonds.

Bis(2-aminopyridinium) hexaaquacopper(II) bis(sulfate) tetrahydrate

\section{Crystal data}

$\left(\mathrm{C}_{5} \mathrm{H}_{7} \mathrm{~N}_{2}\right)_{2}\left[\mathrm{Cu}\left(\mathrm{H}_{2} \mathrm{O}\right)_{6}\right]\left(\mathrm{SO}_{4}\right)_{2} \cdot 4 \mathrm{H}_{2} \mathrm{O}$

$M_{r}=626.07$

Triclinic, $P \overline{1}$

$a=7.115$ (3) $\AA$

$b=8.211(3) \AA$

$c=12.561$ (4) $\AA$

$\alpha=91.83(3)^{\circ}$

$\beta=104.59(3)^{\circ}$

$\gamma=114.57(3)^{\circ}$

$V=638.0(4) \AA^{3}$

\section{Data collection}

Rigaku Oxford Diffraction Xcalibur, Sapphire2 diffractometer

Radiation source: fine-focus sealed X-ray tube, Enhance (Mo) X-ray Source Graphite monochromator Detector resolution: 8.2214 pixels $\mathrm{mm}^{-1}$ $\omega$ scans
$Z=1$

$F(000)=327$

$D_{\mathrm{x}}=1.629 \mathrm{Mg} \mathrm{m}^{-3}$

Mo $K \alpha$ radiation, $\lambda=0.71073 \AA$

Cell parameters from 2602 reflections

$\theta=3.3-27.4^{\circ}$

$\mu=1.10 \mathrm{~mm}^{-1}$

$T=295 \mathrm{~K}$

Block, green

$0.35 \times 0.14 \times 0.13 \mathrm{~mm}$

Absorption correction: multi-scan

(CrysAlis PRO; Rigaku Oxford Diffraction, 2015)

$T_{\min }=0.720, T_{\max }=1.000$

7926 measured reflections

3173 independent reflections

2268 reflections with $I>2 \sigma(I)$

$R_{\text {int }}=0.038$ 
$\theta_{\max }=29.4^{\circ}, \theta_{\min }=3.0^{\circ}$

$h=-9 \rightarrow 6$

Refinement

Refinement on $F^{2}$

Least-squares matrix: full

$R\left[F^{2}>2 \sigma\left(F^{2}\right)\right]=0.044$

$w R\left(F^{2}\right)=0.094$

$S=1.03$

3173 reflections

160 parameters

0 restraints $k=-10 \rightarrow 11$

$l=-15 \rightarrow 17$

Hydrogen site location: mixed

$\mathrm{H}$-atom parameters constrained

$w=1 /\left[\sigma^{2}\left(F_{\mathrm{o}}^{2}\right)+(0.0324 P)^{2}+0.2243 P\right]$

where $P=\left(F_{\mathrm{o}}^{2}+2 F_{\mathrm{c}}^{2}\right) / 3$

$(\Delta / \sigma)_{\max }<0.001$

$\Delta \rho_{\max }=0.36$ e $\AA^{-3}$

$\Delta \rho_{\min }=-0.41$ e $\AA^{-3}$

Special details

Geometry. All esds (except the esd in the dihedral angle between two 1.s. planes) are estimated using the full covariance matrix. The cell esds are taken into account individually in the estimation of esds in distances, angles and torsion angles; correlations between esds in cell parameters are only used when they are defined by crystal symmetry. An approximate (isotropic) treatment of cell esds is used for estimating esds involving 1.s. planes.

Fractional atomic coordinates and isotropic or equivalent isotropic displacement parameters $\left(\AA^{2}\right)$

\begin{tabular}{|c|c|c|c|c|}
\hline & $x$ & $y$ & $z$ & $U_{\text {iso }} * / U_{\text {eq }}$ \\
\hline $\mathrm{Cu} 1$ & 0.5000 & 0.5000 & 0.5000 & $0.03035(15)$ \\
\hline O11W & 0.1945 & $0.4686(3)$ & $0.35088(16)$ & $0.0476(5)$ \\
\hline H11A & 0.1489 & 0.5468 & 0.3566 & $0.071 *$ \\
\hline H11B & 0.0825 & 0.3704 & 0.3272 & $0.071 *$ \\
\hline O12W & $0.3772(3)$ & $0.2434(2)$ & $0.50851(15)$ & $0.0482(6)$ \\
\hline H12A & 0.3051 & 0.1679 & 0.4500 & $0.072 *$ \\
\hline H12B & 0.3718 & 0.1983 & 0.5673 & $0.072 *$ \\
\hline O13W & 0.3680 & $0.5392(2)$ & $0.61353(14)$ & $0.0359(4)$ \\
\hline H13A & 0.3521 & 0.6347 & 0.6187 & $0.054 *$ \\
\hline H13B & 0.2510 & 0.4506 & 0.6110 & $0.054 *$ \\
\hline $\mathrm{S} 1$ & $0.34958(11)$ & $-0.04901(9)$ & $0.72795(5)$ & 0.03015 (17) \\
\hline O11 & $0.5437(3)$ & $-0.0526(3)$ & $0.80214(15)$ & $0.0434(5)$ \\
\hline $\mathrm{O} 12$ & 0.1755 & $-0.1167(3)$ & $0.78078(18)$ & $0.0541(6)$ \\
\hline $\mathrm{O} 13$ & $0.3945(4)$ & $0.1366(3)$ & $0.70846(16)$ & $0.0493(6)$ \\
\hline $\mathrm{O} 14$ & $0.2833(4)$ & -0.1658 & $0.62203(16)$ & $0.0566(6)$ \\
\hline N1 & $0.6656(4)$ & 0.4231 & $0.89059(17)$ & $0.0385(6)$ \\
\hline H1 & 0.6136 & 0.3421 & 0.8326 & $0.046^{*}$ \\
\hline N2 & 0.6983 (4) & 0.2085 & 0.99866 (19) & $0.0462(6)$ \\
\hline $\mathrm{H} 2 \mathrm{~A}$ & 0.6458 & 0.1297 & 0.9395 & $0.055^{*}$ \\
\hline $\mathrm{H} 2 \mathrm{~B}$ & 0.7349 & 0.1784 & 1.0629 & $0.055^{*}$ \\
\hline $\mathrm{C} 2$ & $0.7227(4)$ & $0.3756(4)$ & $0.9910(2)$ & $0.0364(6)$ \\
\hline $\mathrm{C} 3$ & $0.8066(5)$ & $0.5115(4)$ & $1.0846(2)$ & $0.0484(8)$ \\
\hline $\mathrm{H} 3$ & 0.8482 & 0.4852 & 1.1557 & $0.058 *$ \\
\hline $\mathrm{C} 4$ & $0.8263(5)$ & $0.6786(4)$ & $1.0711(3)$ & $0.0527(8)$ \\
\hline $\mathrm{H} 4$ & 0.8812 & 0.7670 & 1.1334 & $0.063 *$ \\
\hline $\mathrm{C} 5$ & $0.7659(5)$ & $0.7227(4)$ & $0.9654(3)$ & $0.0510(8)$ \\
\hline H5 & 0.7806 & 0.8391 & 0.9566 & $0.061^{*}$ \\
\hline C6 & $0.6855(5)$ & $0.5915(4)$ & 0.8760 & $0.0477(8)$ \\
\hline
\end{tabular}




\begin{tabular}{lllll} 
H6 & 0.6441 & 0.6171 & 0.8046 & $0.057^{*}$ \\
O14W & $-0.0364(3)$ & $0.2860(3)$ & $0.59552(16)$ & $0.0432(5)$ \\
H14A & -0.0658 & 0.1958 & 0.6291 & $0.065^{*}$ \\
H14B & -0.1086 & 0.2458 & 0.5285 & $0.065^{*}$ \\
O15W & $0.1567(3)$ & $0.0248(2)$ & $0.30825(15)$ & $0.0381(5)$ \\
H15A & 0.2371 & 0.0360 & 0.2675 & $0.057^{*}$ \\
H15B & 0.0585 & 0.0513 & 0.2734 & $0.057^{*}$ \\
\hline
\end{tabular}

Atomic displacement parameters $\left(\AA^{2}\right)$

\begin{tabular}{lllllll}
\hline & $U^{11}$ & $U^{22}$ & $U^{33}$ & $U^{12}$ & $U^{13}$ & $U^{23}$ \\
\hline Cu1 & $0.0418(3)$ & $0.0233(2)$ & $0.0246(2)$ & $0.0126(2)$ & $0.0103(2)$ & $0.00304(17)$ \\
O11W & $0.0435(13)$ & $0.0422(12)$ & $0.0502(13)$ & $0.0187(10)$ & $0.0034(10)$ & $-0.0031(9)$ \\
O12W & $0.0811(16)$ & $0.0242(10)$ & $0.0267(10)$ & $0.0126(10)$ & $0.0132(10)$ & $0.0046(8)$ \\
O13W & $0.0416(11)$ & $0.0323(10)$ & $0.0345(10)$ & $0.0166(9)$ & $0.0121(9)$ & $0.0024(8)$ \\
S1 & $0.0370(4)$ & $0.0289(4)$ & $0.0247(3)$ & $0.0156(3)$ & $0.0071(3)$ & $0.0055(3)$ \\
O11 & $0.0407(12)$ & $0.0640(14)$ & $0.0317(10)$ & $0.0304(11)$ & $0.0077(9)$ & $0.0081(9)$ \\
O12 & $0.0433(13)$ & $0.0771(16)$ & $0.0562(14)$ & $0.0330(12)$ & $0.0231(11)$ & $0.0360(12)$ \\
O13 & $0.0755(16)$ & $0.0315(11)$ & $0.0321(11)$ & $0.0188(11)$ & $0.0090(10)$ & $0.0076(8)$ \\
O14 & $0.0838(17)$ & $0.0504(13)$ & $0.0325(11)$ & $0.0404(13)$ & $-0.0057(11)$ & $-0.0096(9)$ \\
N1 & $0.0453(15)$ & $0.0451(15)$ & $0.0225(11)$ & $0.0202(12)$ & $0.0053(10)$ & $0.0028(10)$ \\
N2 & $0.0582(17)$ & $0.0452(15)$ & $0.0291(12)$ & $0.0205(13)$ & $0.0062(12)$ & $0.0065(10)$ \\
C2 & $0.0369(17)$ & $0.0448(17)$ & $0.0254(14)$ & $0.0166(14)$ & $0.0081(12)$ & $0.0043(11)$ \\
C3 & $0.051(2)$ & $0.057(2)$ & $0.0256(14)$ & $0.0178(17)$ & $0.0040(14)$ & $0.0018(13)$ \\
C4 & $0.052(2)$ & $0.050(2)$ & $0.0408(18)$ & $0.0150(17)$ & $0.0032(15)$ & $-0.0090(15)$ \\
C5 & $0.049(2)$ & $0.0418(18)$ & $0.058(2)$ & $0.0192(16)$ & $0.0097(17)$ & $0.0062(15)$ \\
C6 & $0.051(2)$ & $0.055(2)$ & $0.0384(17)$ & $0.0273(17)$ & $0.0093(15)$ & $0.0133(14)$ \\
O14W & $0.0480(13)$ & $0.0359(11)$ & $0.0390(11)$ & $0.0149(10)$ & $0.0075(10)$ & $0.0070(8)$ \\
O15W & $0.0356(11)$ & $0.0438(12)$ & $0.0357(10)$ & $0.0190(9)$ & $0.0090(9)$ & $0.0048(8)$ \\
& & & & & & \\
\hline
\end{tabular}

Geometric parameters $\left(\AA,{ }^{\circ}\right)$

\begin{tabular}{llll}
\hline $\mathrm{Cu} 1-\mathrm{O} 11 \mathrm{~W}$ & $2.398(2)$ & $\mathrm{N} 1-\mathrm{C} 2$ & $1.347(3)$ \\
$\mathrm{Cu} 1-\mathrm{O} 11 \mathrm{~W}^{\mathrm{i}}$ & $2.398(2)$ & $\mathrm{N} 1-\mathrm{C} 6$ & $1.353(4)$ \\
$\mathrm{Cu} 1-\mathrm{O} 12 \mathrm{~W}^{\mathrm{i}}$ & $1.935(2)$ & $\mathrm{N} 2-\mathrm{H} 2 \mathrm{~A}$ & 0.8600 \\
$\mathrm{Cu} 1-\mathrm{O} 12 \mathrm{~W}$ & $1.935(2)$ & $\mathrm{N} 2-\mathrm{H} 2 \mathrm{~B}$ & 0.8600 \\
$\mathrm{Cu} 1-\mathrm{O} 13 \mathrm{~W}$ & $1.9790(18)$ & $\mathrm{N} 2-\mathrm{C} 2$ & $1.319(4)$ \\
$\mathrm{Cu} 1-\mathrm{O} 13 \mathrm{~W}^{\mathrm{i}}$ & $1.9790(18)$ & $\mathrm{C} 2-\mathrm{C} 3$ & $1.412(4)$ \\
$\mathrm{O} 11 \mathrm{~W}-\mathrm{H} 11 \mathrm{~A}$ & 0.8397 & $\mathrm{C} 3-\mathrm{H} 3$ & 0.9300 \\
$\mathrm{O} 11 \mathrm{~W}-\mathrm{H} 11 \mathrm{~B}$ & 0.8396 & $\mathrm{C} 3-\mathrm{C} 4$ & $1.341(4)$ \\
$\mathrm{O} 12 \mathrm{~W}-\mathrm{H} 12 \mathrm{~A}$ & 0.8396 & $\mathrm{C} 4-\mathrm{H} 4$ & 0.9300 \\
$\mathrm{O} 12 \mathrm{~W}-\mathrm{H} 12 \mathrm{~B}$ & 0.8394 & $\mathrm{C} 4-\mathrm{C} 5$ & $1.397(4)$ \\
$\mathrm{O} 13 \mathrm{~W}-\mathrm{H} 13 \mathrm{~A}$ & 0.8398 & $\mathrm{C} 5-\mathrm{H} 5$ & 0.9300 \\
$\mathrm{O} 13 \mathrm{~W}-\mathrm{H} 13 \mathrm{~B}$ & 0.8398 & $\mathrm{C} 5-\mathrm{C} 6$ & $1.355(4)$ \\
$\mathrm{S} 1-\mathrm{O} 11$ & $1.471(2)$ & $\mathrm{C} 6-\mathrm{H} 6$ & 0.9300 \\
$\mathrm{~S} 1-\mathrm{O} 12$ & $1.466(2)$ & $\mathrm{O} 14 \mathrm{~W}-\mathrm{H} 14 \mathrm{~A}$ & 0.8397 \\
$\mathrm{~S} 1-\mathrm{O} 13$ & $1.464(2)$ & $\mathrm{O} 14 \mathrm{~W}-\mathrm{H} 14 \mathrm{~B}$ & 0.8397 \\
$\mathrm{~S} 1-\mathrm{O} 14$ & $1.462(2)$ & $\mathrm{O} 15 \mathrm{~W}-\mathrm{H} 15 \mathrm{~A}$ & 0.8399
\end{tabular}




\begin{tabular}{|c|c|c|c|}
\hline $\mathrm{N} 1-\mathrm{H} 1$ & 0.8600 & $\mathrm{O} 15 \mathrm{~W}-\mathrm{H} 15 \mathrm{~B}$ & 0.8401 \\
\hline $\mathrm{O} 11 \mathrm{~W}-\mathrm{Cu} 1-\mathrm{O} 11 \mathrm{~W}$ & 180.0 & $\mathrm{O} 14-\mathrm{S} 1-\mathrm{O} 11$ & $109.47(12)$ \\
\hline $\mathrm{O} 12 \mathrm{~W}-\mathrm{Cu} 1-\mathrm{O} 11 \mathrm{~W}$ & $92.90(9)$ & $\mathrm{O} 14-\mathrm{S} 1-\mathrm{O} 12$ & $109.29(15)$ \\
\hline $\mathrm{O} 12 \mathrm{~W}^{\mathrm{i}}-\mathrm{Cu} 1-\mathrm{O} 11 \mathrm{~W}^{\mathrm{i}}$ & $92.90(9)$ & $\mathrm{O} 14-\mathrm{S} 1-\mathrm{O} 13$ & $109.78(13)$ \\
\hline $\mathrm{O} 12 \mathrm{~W}^{\mathrm{i}}-\mathrm{Cu} 1-\mathrm{O} 11 \mathrm{~W}$ & $87.10(9)$ & $\mathrm{C} 2-\mathrm{N} 1-\mathrm{H} 1$ & 118.2 \\
\hline $\mathrm{O} 12 \mathrm{~W}-\mathrm{Cu} 1-\mathrm{O} 11 \mathrm{~W}^{\mathrm{i}}$ & $87.10(9)$ & $\mathrm{C} 2-\mathrm{N} 1-\mathrm{C} 6$ & $123.6(2)$ \\
\hline $\mathrm{O} 12 \mathrm{~W}^{\mathrm{i}}-\mathrm{Cu} 1-\mathrm{O} 12 \mathrm{~W}$ & 180.0 & $\mathrm{C} 6-\mathrm{N} 1-\mathrm{H} 1$ & 118.2 \\
\hline $\mathrm{O} 12 \mathrm{~W}-\mathrm{Cu} 1-\mathrm{O} 13 \mathrm{~W}$ & $89.66(8)$ & $\mathrm{H} 2 \mathrm{~A}-\mathrm{N} 2-\mathrm{H} 2 \mathrm{~B}$ & 120.0 \\
\hline $\mathrm{O} 12 \mathrm{~W}^{\mathrm{i}}-\mathrm{Cu} 1-\mathrm{O} 13 \mathrm{~W}^{\mathrm{i}}$ & $89.66(9)$ & $\mathrm{C} 2-\mathrm{N} 2-\mathrm{H} 2 \mathrm{~A}$ & 120.0 \\
\hline $\mathrm{O} 12 \mathrm{~W}^{\mathrm{i}}-\mathrm{Cu} 1-\mathrm{O} 13 \mathrm{~W}$ & $90.34(8)$ & $\mathrm{C} 2-\mathrm{N} 2-\mathrm{H} 2 \mathrm{~B}$ & 120.0 \\
\hline $\mathrm{O} 12 \mathrm{~W}-\mathrm{Cu} 1-\mathrm{O} 13 \mathrm{~W}^{\mathrm{i}}$ & $90.34(8)$ & $\mathrm{N} 1-\mathrm{C} 2-\mathrm{C} 3$ & $116.8(3)$ \\
\hline $\mathrm{O} 13 \mathrm{~W}^{\mathrm{i}}-\mathrm{Cu} 1-\mathrm{O} 11 \mathrm{~W}$ & $88.14(8)$ & $\mathrm{N} 2-\mathrm{C} 2-\mathrm{N} 1$ & $120.1(2)$ \\
\hline $\mathrm{O} 13 \mathrm{~W}-\mathrm{Cu} 1-\mathrm{O} 11 \mathrm{~W}^{\mathrm{i}}$ & $88.14(8)$ & $\mathrm{N} 2-\mathrm{C} 2-\mathrm{C} 3$ & $123.1(3)$ \\
\hline $\mathrm{O} 13 \mathrm{~W}^{\mathrm{i}}-\mathrm{Cu} 1-\mathrm{O} 11 \mathrm{~W}^{\mathrm{i}}$ & $91.86(8)$ & $\mathrm{C} 2-\mathrm{C} 3-\mathrm{H} 3$ & 119.9 \\
\hline $\mathrm{O} 13 \mathrm{~W}-\mathrm{Cu} 1-\mathrm{O} 11 \mathrm{~W}$ & $91.86(8)$ & $\mathrm{C} 4-\mathrm{C} 3-\mathrm{C} 2$ & $120.1(3)$ \\
\hline $\mathrm{O} 13 \mathrm{~W}-\mathrm{Cu} 1-\mathrm{O} 13 \mathrm{~W}^{\mathrm{i}}$ & $180.00(11)$ & $\mathrm{C} 4-\mathrm{C} 3-\mathrm{H} 3$ & 119.9 \\
\hline $\mathrm{Cu} 1-\mathrm{O} 11 \mathrm{~W}-\mathrm{H} 11 \mathrm{~A}$ & 115.8 & $\mathrm{C} 3-\mathrm{C} 4-\mathrm{H} 4$ & 119.3 \\
\hline $\mathrm{Cu} 1-\mathrm{O} 11 \mathrm{~W}-\mathrm{H} 11 \mathrm{~B}$ & 122.9 & $\mathrm{C} 3-\mathrm{C} 4-\mathrm{C} 5$ & $121.3(3)$ \\
\hline $\mathrm{H} 11 \mathrm{~A}-\mathrm{O} 11 \mathrm{~W}-\mathrm{H} 11 \mathrm{~B}$ & 104.7 & $\mathrm{C} 5-\mathrm{C} 4-\mathrm{H} 4$ & 119.3 \\
\hline $\mathrm{Cu} 1-\mathrm{O} 12 \mathrm{~W}-\mathrm{H} 12 \mathrm{~A}$ & 119.8 & $\mathrm{C} 4-\mathrm{C} 5-\mathrm{H} 5$ & 120.9 \\
\hline $\mathrm{Cu} 1-\mathrm{O} 12 \mathrm{~W}-\mathrm{H} 12 \mathrm{~B}$ & 125.3 & $\mathrm{C} 6-\mathrm{C} 5-\mathrm{C} 4$ & $118.2(3)$ \\
\hline $\mathrm{H} 12 \mathrm{~A}-\mathrm{O} 12 \mathrm{~W}-\mathrm{H} 12 \mathrm{~B}$ & 114.2 & $\mathrm{C} 6-\mathrm{C} 5-\mathrm{H} 5$ & 120.9 \\
\hline $\mathrm{Cu} 1-\mathrm{O} 13 \mathrm{~W}-\mathrm{H} 13 \mathrm{~A}$ & 118.5 & $\mathrm{~N} 1-\mathrm{C} 6-\mathrm{C} 5$ & $119.9(3)$ \\
\hline $\mathrm{Cu} 1-\mathrm{O} 13 \mathrm{~W}-\mathrm{H} 13 \mathrm{~B}$ & 113.2 & $\mathrm{~N} 1-\mathrm{C} 6-\mathrm{H} 6$ & 120.0 \\
\hline $\mathrm{H} 13 \mathrm{~A}-\mathrm{O} 13 \mathrm{~W}-\mathrm{H} 13 \mathrm{~B}$ & 108.7 & $\mathrm{C} 5-\mathrm{C} 6-\mathrm{H} 6$ & 120.0 \\
\hline $\mathrm{O} 12-\mathrm{S} 1-\mathrm{O} 11$ & $108.78(12)$ & $\mathrm{H} 14 \mathrm{~A}-\mathrm{O} 14 \mathrm{~W}-\mathrm{H} 14 \mathrm{~B}$ & 106.1 \\
\hline $\mathrm{O} 13-\mathrm{S} 1-\mathrm{O} 11$ & $110.06(13)$ & $\mathrm{H} 15 \mathrm{~A}-\mathrm{O} 15 \mathrm{~W}-\mathrm{H} 15 \mathrm{~B}$ & 106.7 \\
\hline $\mathrm{O} 13-\mathrm{S} 1-\mathrm{O} 12$ & $109.43(13)$ & & \\
\hline $\mathrm{N} 1-\mathrm{C} 2-\mathrm{C} 3-\mathrm{C} 4$ & $0.0(4)$ & $\mathrm{C} 3-\mathrm{C} 4-\mathrm{C} 5-\mathrm{C} 6$ & $0.3(5)$ \\
\hline $\mathrm{N} 2-\mathrm{C} 2-\mathrm{C} 3-\mathrm{C} 4$ & $-179.7(3)$ & $\mathrm{C} 4-\mathrm{C} 5-\mathrm{C} 6-\mathrm{N} 1$ & $0.0(5)$ \\
\hline $\mathrm{C} 2-\mathrm{N} 1-\mathrm{C} 6-\mathrm{C} 5$ & $-0.3(5)$ & $\mathrm{C} 6-\mathrm{N} 1-\mathrm{C} 2-\mathrm{N} 2$ & $179.9(3)$ \\
\hline $\mathrm{C} 2-\mathrm{C} 3-\mathrm{C} 4-\mathrm{C} 5$ & $-0.2(5)$ & $\mathrm{C} 6-\mathrm{N} 1-\mathrm{C} 2-\mathrm{C} 3$ & $0.3(4)$ \\
\hline
\end{tabular}

Symmetry code: (i) $-x+1,-y+1,-z+1$.

Hydrogen-bond geometry $\left(\AA,{ }^{\circ}\right)$

\begin{tabular}{lllll}
\hline$D-\mathrm{H} \cdots A$ & $D-\mathrm{H}$ & $\mathrm{H} \cdots A$ & $D \cdots A$ & $D-\mathrm{H} \cdots A$ \\
\hline $\mathrm{O} 11 W-\mathrm{H} 11 A \cdots \mathrm{O} 14 W^{\mathrm{ji}}$ & 0.84 & 2.00 & $2.821(3)$ & 167 \\
$\mathrm{O} 11 W-\mathrm{H} 11 B \cdots \mathrm{O} 12^{\mathrm{iii}}$ & 0.84 & 2.22 & $3.032(4)$ & 162 \\
$\mathrm{O} 12 W-\mathrm{H} 12 A \cdots \mathrm{O} 15 W$ & 0.84 & 1.88 & $2.719(3)$ & 172 \\
$\mathrm{O} 12 W-\mathrm{H} 12 B \cdots \mathrm{O} 13$ & 0.84 & 1.85 & $2.677(3)$ & 171 \\
$\mathrm{O} 13 W-\mathrm{H} 13 A \cdots \mathrm{O} 14^{\text {iv }}$ & 0.84 & 1.90 & $2.733(3)$ & 174 \\
$\mathrm{O} 13 W-\mathrm{H} 13 B \cdots \mathrm{O} 14 W$ & 0.84 & 1.88 & $2.706(3)$ & 168 \\
$\mathrm{~N} 1-\mathrm{H} 1 \cdots \mathrm{O} 13$ & 0.86 & 2.03 & $2.855(3)$ & 160 \\
$\mathrm{~N} 2-\mathrm{H} 2 A \cdots \mathrm{O} 11$ & 0.86 & 2.01 & $2.869(3)$ & 176
\end{tabular}




$\begin{array}{lllll}\mathrm{N} 2-\mathrm{H} 2 B \cdots \mathrm{O} 12^{\mathrm{v}} & 0.86 & 2.05 & 2.914(3) & 178 \\ \mathrm{O} 14 W-\mathrm{H} 14 A^{\cdots} \mathrm{O} 15 W^{\mathrm{iii}} & 0.84 & 1.92 & 2.758(3) & 174 \\ \mathrm{O} 14 W-\mathrm{H} 14 B \cdots \mathrm{O} 14^{\mathrm{iii}} & 0.84 & 1.90 & 2.738(3) & 176 \\ \mathrm{O} 15 W-\mathrm{H} 15 A \cdots \mathrm{O} 11^{\mathrm{vi}} & 0.84 & 1.93 & 2.761(3) & 169 \\ \mathrm{O} 15 W-\mathrm{H} 15 B \cdots \mathrm{O} 12^{\mathrm{iii}} & 0.84 & 1.93 & 2.760(3) & 170\end{array}$

Symmetry codes: (ii) $-x,-y+1,-z+1$; (iii) $-x,-y,-z+1$; (iv) $x, y+1, z$; (v) $-x+1,-y,-z+2$; (vi) $-x+1,-y,-z+1$. 